\title{
The impact of predictive genetic testing for hereditary nonpolyposis colorectal cancer: three years after testing
}

\author{
Veronica R. Collins, $P h D^{1}$, Bettina Meiser, $P h D^{2}$, Obioha C. Ukoumunne, PhD ${ }^{3}$, Clara Gaff, PhD ${ }^{4,5}$,
} D. James St. John, $M D^{5,6}$, and Jane L. Halliday, $P h D^{1}$

\begin{abstract}
Background: To fully assess predictive genetic testing programs, it is important to assess outcomes over periods of time longer than the 1-year follow-up reported in the literature. Methods: We conducted a 3-year study of individuals who received predictive genetic test results for previously identified familial mutations in Australian Familial Cancer Clinics. Questionnaires were sent before attendance at the familial cancer clinic and 2 weeks, 4 months, 1 year, and 3 years after receiving test results. Psychological measures were included each time, and preventive behaviors were assessed at baseline and 1 and 3 years. Psychological measures were adjusted for age, gender, and baseline score. Results: The study included 19 carriers and 54 non-carriers. We previously reported an increase in mean cancer-specific distress in carriers at 2 weeks with a return to baseline levels by 12 months. This level was maintained until 3 years. Non-carriers showed sustained decreases after testing with a significantly lower level at 3 years compared with baseline $(P<0.001)$. These scores tended to be lower than those for carriers at 3 years $(P=0.09)$. Mean depression and anxiety scores did not differ between carriers and non-carriers and, at 3 years, were similar to baseline. All carriers and 7\% of non-carriers had had a colonoscopy by 3 years, and $69 \%$ of 13 female carriers had undergone gynecological screening in the previous 2 years. Prophylactic surgery was rare. Conclusion: This report of long-term data indicates appropriate screening and improved psychological measures for non-carriers with no evidence of undue psychological distress in carriers of hereditary nonpolyposis colorectal cancer mutations. Genet Med 2007:9(5):290-297.
\end{abstract}

Key Words: Hereditary nonpolyposis colorectal cancer, genetic testing, psychological impact, colonoscopy, endometrial cancer

\section{INTRODUCTION}

The past decade has seen predictive genetic testing for familial cancers move from the research setting into clinical practice, so that genetic testing is now an integral part of familial cancer services in many countries. An ethical requirement of offering predictive genetic testing as a clinical service is to ensure that the provision of genetic risk information is not un-

From the ${ }^{1}$ Public Health Genetics, Murdoch Childrens Research Institute, Victoria; ${ }^{2}$ Psychosocial Research Group, Department of Medical Oncology, Prince of Wales Hospital, and School of Psychiatry, University of New South Wales, New South Wales; ${ }^{3}$ Clinical Epidemiology and Biostatistics Unit, Murdoch Childrens Research Institute and Department of Paediatrics, University of Melbourne, Victoria; ${ }^{4}$ Genetic Health Services Victoria, and Royal Children's Hospital, Victoria; ${ }^{5}$ Familial Cancer Centre, The Royal Melbourne Hospital, Victoria; ${ }^{6}$ National Cancer Control Initiative, Melbourne, Victoria, Australia.

Veronica Collins, PhD, Public Health Genetics, Murdoch Childrens Research Institute, 10th Floor, Royal Children's Hospital, Flemington Rd., Parkville, VIC 3052, Australia. Email: veronica.collins@mcri.edu.au

The authors declare no conflict of interest.

Submitted for publication October 19, 2006

Accepted for publication February 6, 2006

DOI: 10.1097/GIM.0b013e31804b45db duly detrimental to psychological well-being, that it is understood by patients, and that it is associated with appropriate screening and surveillance behavior. Research on the effectiveness of testing programs and the impact of receiving genetic information is therefore an integral part of the development and ongoing monitoring of genetic testing programs in familial cancers.

Hereditary nonpolyposis colorectal cancer (HNPCC) is a familial cancer syndrome that accounts for $1 \%$ to $3 \%$ of all colorectal cancers (CRC). ${ }^{1,2}$ Several mismatch repair genes have been identified in which mutations have been shown to predispose a person to HNPCC. ${ }^{3}$ In addition to CRC, HNPCC also includes cancers of other tissues, particularly the endometrium and ovaries in women. ${ }^{4,5}$ Predictive genetic testing in families affected by HNPCC has been available since $1994 .{ }^{6,7}$ Early family-based studies that did not adjust for the source of ascertainment of cancer cases indicated a very high lifetime risk of colorectal cancer for men and a lower risk for women. ${ }^{4}$ However, more recent studies suggest the risk is not quite as high. ${ }^{8-10}$ One Australian population-based study has estimated the risk of CRC up to age 70 years as $45 \%$ for men and $38 \%$ for women, and for any HNPCC-related cancer, $67 \%$ for men and $72 \%$ for women. ${ }^{8}$ 
The potential benefits of predictive genetic testing for HNPCC have been highlighted by studies demonstrating the efficacy of colonoscopy screening in reducing the incidence of, and mortality from, colorectal cancer. ${ }^{11,12}$ Moreover, the potential benefits for the health system have been modeled in an Australian population in which substantial cost-savings were demonstrated if targeted surveillance in mutation carriers and population-level surveillance in non-carriers were adopted after testing. ${ }^{13}$

Until now, studies of screening behaviors and the psychological impact of predictive genetic testing for HNPCC have only reported follow-up periods of 1 year, including two publications from our cohort. ${ }^{14,15}$ It is currently unknown whether the generally reassuring results reported in these studies continue in the longer term. Herein we report on psychological measures and screening behaviors in both carriers and noncarriers of mutations predisposing to HNPCC, 3 years after receiving test results.

\section{SUBJECTS AND METHODS}

The methods for this study have been published elsewhere, along with reported outcomes of the 12-month follow-up survey, ${ }^{14,15}$ and will be outlined briefly herein, together with details specific to the 3-year follow-up.

\section{Predictive genetic testing}

In Australia, predictive genetic testing should only be offered to family members once a family-specific pathogenic HNPCC mutation has been identified in an individual affected by cancer. ${ }^{16}$ The standard protocol for undergoing predictive genetic testing in Australia is to attend a familial cancer service for at least one session of genetic counseling before the collection of blood. ${ }^{17}$ The predictive test result is then given to the individual within a genetic counseling protocol, usually by the person who has conducted the initial counseling. Screening recommendations, based on the test result and any other specific medical details, are given by the relevant medical specialist. Letters outlining the recommended screening are then sent to the individuals concerned, as well as to other practitioners involved in their care.

\section{Screening recommendations}

The Australian guidelines for the prevention, early detection, and management of CRC form the basis of screening recommendations for those at risk of HNPCC. ${ }^{18}$ Annual colonoscopy from the age of 25 years (or 5 years younger than the youngest affected family member) is recommended for those found to carry a mutation. Those not carrying a mutation are advised to follow population guidelines; that is, annual or biennial fecal occult blood testing (FOBT) with the possibility of 5-yearly sigmoidoscopy. Because of the lack of proven benefit, the guidelines for endometrial or ovarian cancer screening in women carrying a mutation for HNPCC are not as prescriptive as those for colorectal screening. It is suggested that women carrying a mutation consider endometrial screening beginning between the ages of 30 and 35 years. ${ }^{18}$ The familial cancer clinics generally recommend annual transvaginal ultrasound and endometrial sampling in premenopausal women, and annual transvaginal ultrasound and CA125 testing for postmenopausal women. Preventive colectomy is not routinely recommended for those carrying a mutation but may be discussed as an option, depending on individual circumstances. For women carrying a mutation, prophylactic hysterectomy and oophorectomy might also be considered after the age of 30 to 35 years or when childbearing is complete. ${ }^{18}$

\section{Participants}

The sample included individuals undergoing predictive genetic testing at one of five familial cancer clinics in Australia between June 1998 and August 2002. The study was approved by five institutional ethics committees, and participants gave signed informed consent. Only individuals who had never had CRC or any of the cancers associated with HNPCC were included. Staff of the familial cancer clinics invited individuals to participate in the study during the pre-clinic telephone call. All subsequent correspondence was via the coordinating research center. Data were collected at five time points by self-completed questionnaires mailed to participants. Baseline questionnaires were sent to participants before they first attended the familial cancer clinic, having been invited to participate in the study during the pre-clinical telephone call. Follow-up questionnaires were sent 2 weeks, 4 months, 12 months, and 3 years after receiving test results. Before sending the 3-year questionnaire, up-to-date addresses were sought from the telephone directory, the electoral roll, or the clinics. Participants known to have died or developed cancer in the 3 years since genetic testing were not sent a questionnaire. Further to this, a form was included with the 3-year questionnaire to ascertain whether the participant had developed cancer; if so, they were asked to return the form without completing the questionnaire.

\section{Measures}

\section{Demographic characteristics}

At baseline, age, sex, educational level, marital status, and number of children were recorded.

\section{Mutation carrier risk}

To provide an estimate of risk before genetic testing, individuals were given a $50 \%, 25 \%, 12.5 \%$, or $6.25 \%$ risk of carrying a mutation based on their relationship to the person in whom the mutation had been identified.

\section{Screening behaviors}

Information on colonoscopic and FOBT screening was collected by self-report at baseline and 12 months and 3 years after receiving a predictive genetic test result. Women were asked similar questions regarding transvaginal ultrasound or endometrial sampling for endometrial or ovarian cancer screening. 


\section{Prophylactic surgery}

At 12 months and 3 years follow-up, participants were asked whether a prophylactic colectomy had been performed or if they intended to have surgery. Women were asked similar questions regarding prophylactic hysterectomy and bilateral oophorectomy.

\section{Beliefs about screening}

Questions to assess beliefs about bowel cancer and effectiveness of screening were devised based on constructs postulated by the Health Belief Model and Self-Motivation Theory, previously used in a model to describe adherence to CRC screening. ${ }^{19}$ In the 3-year questionnaire, these questions were included to assess motivations for undergoing or not undergoing screening in the previous 2 years (since the 12-month survey). Women were asked the same questions in relation to endometrial cancer.

\section{Psychological measures}

Three validated psychological measures were included at each time point.

\section{Depression scale of the hospital anxiety and depression scale (HADS)}

The HADS is a widely used measure of emotional disturbance. The seven-item Depression Scale of the HADS has been shown to possess high internal consistency, and separate use of its scales is justified. ${ }^{20}$ Scores range from 0 to $21 .^{21}$

\section{State component of the state-trait anxiety inventory - short version (STAI-State)}

A previously validated, prorated six-item short version of the State scale of the Spielberger State-Trait Anxiety Inventory was included as a measure of situational anxiety. ${ }^{22}$ This short version produces scores similar to those obtained using the full 20 -item version, ranging from 20 to 80. It has acceptable reliability and validity and has been used in related studies (e.g., Michie et al. ${ }^{23}$ ).

\section{Impact of event scale (IES)}

This is a 15 -item validated scale that measures intrusive and avoidant thoughts about a specific stressful event ${ }^{24}$ and has been validated for use with women with a family history of breast cancer. ${ }^{25}$ In the current study, the particular stressor was concern about being at risk of developing CRC. Total scores (range 0-75) were used in the analysis.

\section{Statistical analysis}

Data were analyzed using SPSS 11.5 (Statistical Program for the Social Sciences) and Stata 9 (Stata Corporation, 2005). The characteristics of responders to the 3-year questionnaire were compared with those of non-responders. The $\chi^{2}$ test or Fisher's exact test (used when expected cell frequencies were $<5$ ) was used to compare categorical variables; quantitative variables were compared using the Mann-Whitney test or the t-test, as appropriate and indicated in Tables 1 to 3. The characteristics of the subjects are also reported by carrier versus non-carrier status.

For the psychological outcome measures, within-group and between-group analyses were performed. The unadjusted mean levels (with 95\% confidence intervals) of each measure at each time point are presented in Figures 1 to 3. Adjusted anal-

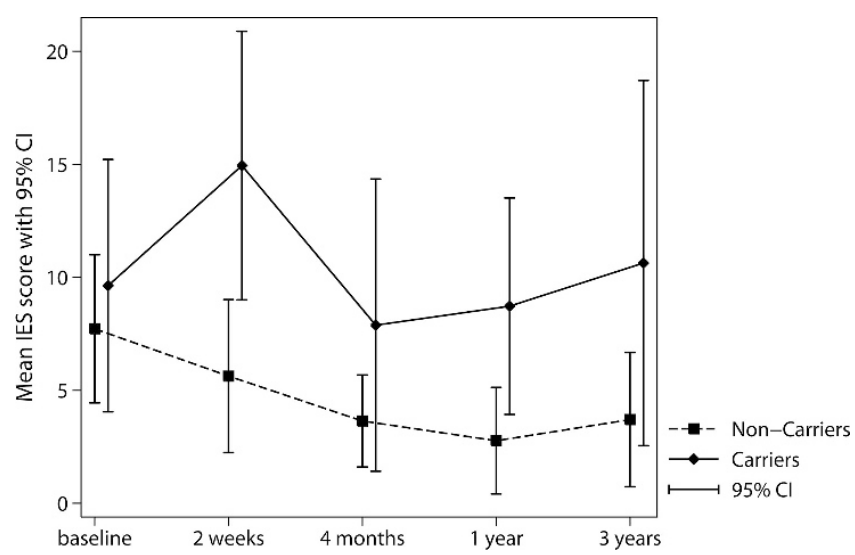

Fig. 1. Mean raw score with $95 \%$ confidence intervals (CI) for cancer-specific distress (IES) at each time point for carriers and non-carriers.

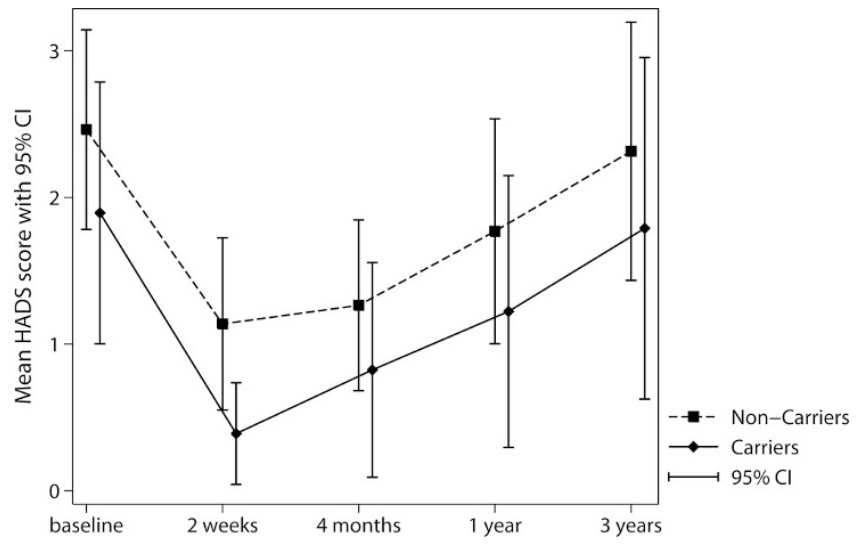

Fig. 2. Mean raw score with $95 \%$ confidence intervals (CI) for depression (HADS) at each time point for carriers and non-carriers.

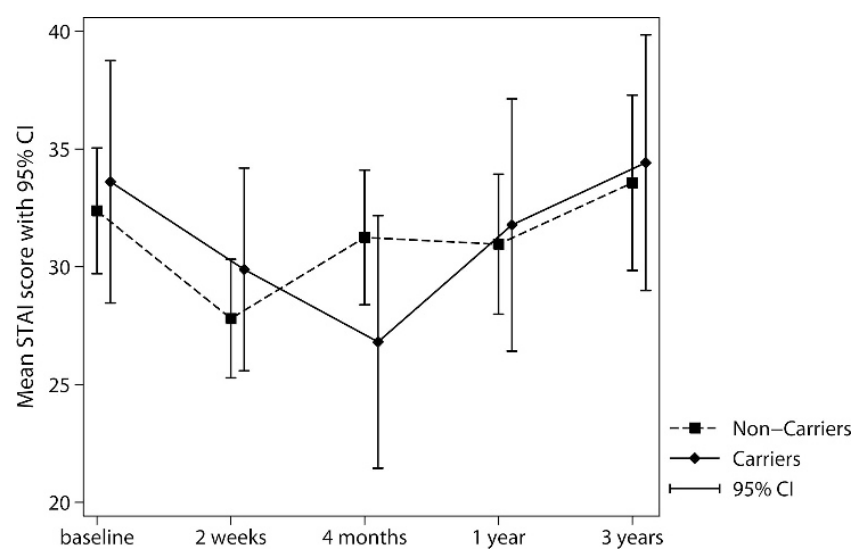

Fig. 3. Mean raw score with $95 \%$ confidence intervals (CI) for State anxiety (STAI) at each time point for carriers and non-carriers. 
yses of the results up to and including the 12-month follow-up have been reported previously. ${ }^{14}$ We therefore summarize these previous findings in "Results" and present the 3-year data that were analyzed using the following methods.

\section{Within-group analyses}

The mean change from baseline to 3 years for each psychological variable was estimated separately for carriers and noncarriers using linear regression. In the model, the baseline and follow-up responses were analyzed as one outcome variable with time (3 years versus baseline), and the potential confounders, age, sex, and pretest risk of being a mutation carrier were used as covariates. Random effects models were fitted ${ }^{26}$ to allow for the correlation between the baseline and 3-year responses from the same subject. This analysis facilitates the estimation of change in a single group while adjusting for confounders.

\section{Between-group analyses}

The analysis of covariance approach was used to compare the mean change in the psychological outcomes from baseline to 3 years between the carriers and non-carriers, adjusting for the potential confounding factors. In the regression model, the follow-up score was used as the outcome, with the baseline score and the potential confounders used as covariates.

As some of the psychological outcomes were skewed, the bootstrap method ${ }^{27}$ was used to validate the confidence intervals from these analyses. The bootstrap confidence intervals were similar to the confidence intervals from the main analyses; therefore, the latter are presented.

\section{RESULTS}

\section{Response to the three-year follow-up questionnaire}

Of the initial 114 participants who completed a baseline questionnaire and received a genetic test result, 2 individuals had died and 5 had developed CRC before the 3-year followup. They were not sent a questionnaire. Another seven participants had actively withdrawn from the study, leaving 100 eligible to receive a 3-year follow-up questionnaire. From this group of 100, up-to-date addresses could not be found for 9 individuals, and 18 did not return the questionnaire. Seventythree questionnaires were completed, corresponding to $64 \%$ of those with baseline data.

Table 1 shows the baseline characteristics of those included in the 3-year follow-up compared with those who did not complete the follow-up questionnaire and the group who had died or developed cancer. Data are shown for the three groups, but $P$ values relate to comparisons between the responder group $(n=73)$ and the combined group of non-response categories $(n=41)$. The main difference between groups was that those completing the follow-up survey had lower mean levels of cancer-specific distress at baseline (measured by the Impact of Event Scale, $P=0.004$ ).

\section{Characteristics of participants}

Of the 73 participants in the 3 -year follow-up study, 19 were carriers of a mutation and 54 were non-carriers (Table 2). The ages of the study participants ranged from 21 to 75 years at the follow-up, and the carriers tended to be slightly younger. Approximately half of the participants had a family history that included endometrial and/or ovarian cancers in addition to CRC.

Table 1

Baseline characteristics of participants and non-participants in the 3-year follow-up

\begin{tabular}{|c|c|c|c|c|}
\hline & \multicolumn{3}{|c|}{ 3-year follow-up status } & \multirow[b]{2}{*}{$P$ value ${ }^{a}$} \\
\hline & $\begin{array}{l}\text { Completed 3-year } \\
\text { follow-up }\end{array}$ & $\begin{array}{l}\text { Non-responder or no } \\
\text { address found }\end{array}$ & $\begin{array}{l}\text { Died or developed } \\
\text { cancer }\end{array}$ & \\
\hline Number of individuals & 73 & 34 & 7 & \\
\hline Age (yr) (median [IQR]) & $41(17)$ & $35(24)$ & $36(23)$ & 0.17 \\
\hline Sex (\% female $)$ & 62 & 56 & 71 & 0.75 \\
\hline Genetic status (\% carriers) & 26 & 24 & 71 & 0.52 \\
\hline $\begin{array}{l}\text { Intrusive and avoidant thoughts about } \\
\text { cancer (median [IQR] score IES) }\end{array}$ & $3(12)$ & $9(23)$ & $21(17)$ & 0.004 \\
\hline Depression (median [IQR] score HADS) & $2(2)$ & $2(2)$ & $1(2)$ & 0.67 \\
\hline Anxiety (median [IQR] score STAI) & $30(13)$ & $33(10)$ & $37(20)$ & 0.31 \\
\hline $\begin{array}{l}\text { Perceived chance of developing Cancer } \\
\quad(\text { mean }[\mathrm{SD}] \text { score }[1-100])\end{array}$ & $45.4(20.6)$ & $48.9(18.3)$ & $55.7(13.9)$ & 0.22 \\
\hline $\begin{array}{l}\text { Bowel screening (\% had at least one } \\
\text { colonoscopy before baseline) }\end{array}$ & 67 & 64 & 100 & 0.75 \\
\hline
\end{tabular}

${ }^{a}$ Differences between the responder group $(n=73)$ and the combined non-responder groups $(n=41)$ using the Mann-Whitney $U$ test for age, IES score, HADS score and STAI score; $\chi^{2}$ tests for categorical data; and $t$ test for comparison of mean score of perceived chance of developing cancer.

$\mathrm{IQR}$, interquartile range; SD, standard deviation. 
Table 2

Characteristics of three-year follow-up participants according to carrier status

\begin{tabular}{lcccc}
\hline & \multicolumn{2}{c}{ Carriers } & \multicolumn{2}{c}{ Non-carriers } \\
\hline$N$ & \multicolumn{2}{c}{19} & \multicolumn{2}{c}{54} \\
Age (yr) & \multicolumn{2}{c}{$40(23-68)$} & \multicolumn{2}{c}{$46(21-75)$} \\
Gender (female) & 13 & $(68)$ & 32 & $(59)$ \\
Married/de facto & 12 & $(63)$ & 46 & $(85)$ \\
Have children & 13 & $(68)$ & 45 & $(83)$ \\
Post-school education & 14 & $(78)$ & 38 & $(70)$ \\
$\quad$ including trade ed.) & & & & \\
Family history of cancer & & & & \\
$\quad$ Colorectal only & 9 & $(47)$ & 29 & $(54)$ \\
$\quad \begin{array}{l}\text { Endometrial included } \\
\quad \text { Ovarian included }\end{array}$ & 8 & $(42)$ & 16 & $(30)$ \\
$\quad$ Endometrial and & 2 & $(11)$ & 4 & $(7)$ \\
$\quad$ ovarian included & 0 & $(0)$ & 5 & $(9)$ \\
\hline
\end{tabular}

Values are expressed as median (range) for age and all other variables as $n$ (\%).

\section{Psychological response to genetic testing: Within-group analyses}

Figures 1 to 3 show the mean raw scores for the three psychological measures at each time point. As previously reported, cancer-specific distress (IES) was increased for carriers 2 weeks postnotification compared with baseline $(P=0.014)$ but decreased to approximately baseline levels by 4 months and 12 months ${ }^{14}$ (Fig. 1). The data reported herein show that this level was maintained until the 3-year follow-up (adjusted mean change score from baseline $1.0 ; 95 \% \mathrm{CI},-4.0$ to $6.0 ; P=0.69)$. For non-carriers, mean IES scores declined from baseline to 12 months $(P<$ $0.001) .{ }^{14}$ The 3-year data show that this decrease was maintained in the longer term (adjusted mean change score from baseline -4.0 ; $95 \% \mathrm{CI},-6.2$ to $-1.8 ; P<0.001)$.

For measures of depression (Fig. 2), the decreases in mean HADS score in carriers 2 weeks $(P<0.001)$ and 4 months $(P=$ $0.002)$ post-notification were no longer evident at 12 months $(P=0.67) .{ }^{14}$ These data show that depression scores had returned to baseline levels again by 3 years (adjusted mean change score from baseline $-0.1 ; 95 \% \mathrm{CI},-1.0$ to $0.7 ; P=0.81$ ). With respect to non-carriers, the decrease in HADS score reported at 2 weeks, 4 months, and 12 months post-notification ${ }^{14}$ were not maintained at 3 years, at which time scores were similar to baseline levels (adjusted mean change score $-0.1 ; 95 \% \mathrm{CI},-0.7$ to $0.4 ; P=0.63$ ).

For State anxiety (Fig. 3), there was a decrease in mean score at 2 weeks in non-carriers, ${ }^{14}$ and the 3 -year data show anxiety levels similar to baseline for carriers (adjusted mean change score from baseline $0.5 ; 95 \% \mathrm{CI},-4.0$ to $4.9 ; P=0.84$ ) and non-carriers (adjusted mean change score from baseline $0.9 ; 95 \% \mathrm{CI},-2.3$ to $4.2 ; P=0.57)$.

\section{Psychological response to genetic testing: Between-group analyses}

We have previously reported that, compared with non-carriers, carriers had higher cancer-specific distress (mean IES) up until 12 months $(P=0.014)$ after test result notification. ${ }^{14}$ Our 3-year data also show a trend for differences between groups in the same direction (adjusted mean difference 5.0; 95\% CI, -0.74 to $10.7 ; P=0.09$ ). There was no evidence of any other differences between carriers and non-carriers for state anxiety or depression at any of the time points, including 3 years, except that carriers had higher State anxiety 2 weeks post-notification $(P=0.006) .{ }^{14}$

\section{Preventive behaviors}

\section{Colonoscopy}

All carriers $(n=19)$ reported having had a colonoscopy between 12 months and 3 years after receipt of test results. Of the carriers, $72 \%$ had also had a colonoscopy within 12 months of receiving their test result. Four non-carriers (7.4\%) reported having a colonoscopy on the 3-year survey, but none of these four had had a colonoscopy between receiving their test result and the 12-month follow-up. All four were older than 50 years, and two reported an indication for colonoscopy that was not related to their carrier status.

The reason most commonly endorsed as important in the decision to have a colonoscopy was a belief that their risk for developing cancer was high for both carriers and non-carriers (Table 3). Most also endorsed the belief that colonoscopy was effective in finding cancer early and was an important reason for having colonoscopy.

\section{Fecal occult blood testing}

To assess population level colorectal cancer screening among non-carriers, we assessed the use of FOBT in those older than 51 years (to allow time to take up screening once reaching the age of 50, the age suggested in the guidelines). Of this group of 18 participants, $4(22.2 \%)$ reported having had FOBT in the previous 2 years at the 3 -year follow-up; 2 had also had a colonoscopy, as reported above. One other person (5.6\%) reported having FOBT since having received the test result, at the 12-month follow-up, but not at 3 years. It seems that the other 13 non-carriers in this age group had not had FOBT since receiving their test result. In the younger age group ( $\leq 51$ years of age, $n=36$ ), three people in their forties $(8.3 \%)$

Table 3

Reasons for having colonoscopy

\begin{tabular}{lcccc}
\hline & \multicolumn{4}{c}{$\begin{array}{c}\text { Percentage choosing "Somewhat" or } \\
\text { "Definitely Important" }\end{array}$} \\
\cline { 2 - 5 } $\begin{array}{l}\text { Importance of factor in } \\
\text { influencing decision to } \\
\text { have colonoscopy }\end{array}$ & $\begin{array}{c}\text { Carriers } \\
(n=19)\end{array}$ & $\begin{array}{c}\text { Non-carriers } \\
(n=4)\end{array}$ \\
\hline $\begin{array}{l}\text { Believe risk for cancer } \\
\text { is high }\end{array}$ & 18 & $(95)$ & 4 & $(100)$ \\
$\begin{array}{l}\text { Believe gene tests are } \\
\text { inaccurate }\end{array}$ & 3 & $(16)$ & 2 & $(50)$ \\
$\begin{array}{l}\text { Wanted reassurance } \\
\text { Colonoscopy effective }\end{array}$ & 15 & $(79)$ & 3 & $(75)$ \\
Easy to do & 19 & $(100)$ & 3 & $(75)$ \\
\hline
\end{tabular}

Values are expressed as $n(\%)$. 
reported FOBT testing in the previous 2 years at the 3-year follow-up.

\section{Gynecological screening}

Of the 13 female carriers who completed the 3-year follow-up questionnaire, $9(69 \%)$ reported having had transvaginal ultrasound, and 7 (54\%) reported having undergone endometrial sampling in the previous 2 years. Of the four who did not report any gynecological screening during the previous 2 years, two reported having had a hysterectomy and oophorectomy and were thus not eligible for these tests; the third reported having had transvaginal ultrasound on the 1-year follow-up questionnaire; and the fourth was only 23 years of age and therefore unlikely to have been advised to have screening at this time. Of the 32 non-carriers, 2 women reported having had transvaginal ultrasound and one other reported having undergone endometrial sampling in the previous 2 years.

Carriers rated the importance of various reasons for having gynecological screening. All indicated that a belief that they were at high risk of cancer was "somewhat important" or "definitely important" in their decision to have screening. The statement that "screening is effective in finding cancer early" was endorsed as "definitely important" by all carriers. Eight of the nine carriers (89\%) also indicated that "wanting reassurance" and thinking screening was an "easy thing for them to do" were somewhat or definite important influences on their decision.

\section{Prophylactic surgery}

No carriers reported having had a prophylactic colectomy at any point since receiving their genetic test result. Two women, both of whom had had a hysterectomy before the baseline questionnaire, reported having had prophylactic oophorectomy within the 12 months after receipt of their test result. No other women reported having undergone prophylactic hysterectomy or oophorectomy on their 3-year questionnaire.

\section{DISCUSSION}

The findings from this study, the first to report 3-year longitudinal data after predictive genetic testing for HNPCC, suggest that individuals are generally not experiencing major distress and are undergoing screening appropriate to their mutation status. This suggests that immediate and short-term responses to mutation test results reported for this cohort ${ }^{14,15}$ and others ${ }^{28-32}$ are likely to continue in the longer term.

\section{Psychological outcomes}

Our results up to 12 months' follow-up were similar to those of other studies, although some studies have used different measures of distress or anxiety. ${ }^{28-30}$ Non-carriers typically experience a reduction in colon cancer-specific distress, generalized anxiety, or depression for periods of up to 1 year after receipt of test results. ${ }^{14,28-30}$ Findings for mutation carriers generally show an increase in either generalized anxiety ${ }^{28}$ or colon cancer-specific distress ${ }^{14}$ or worries ${ }^{30}$ in the period im- mediate after receipt of test result, with decreased levels again by 1 year after test results. ${ }^{14,28-30}$ Our 3-year data suggest that the level of distress in carriers, which at 12 months was similar to baseline, does not increase again over a longer time period. The mean scores on the HADS depression measure suggest very low levels of depression in this cohort, with little difference between groups. Both groups showed a reduction in mean depression scores in the short-term after receiving test results, but these had returned to baseline levels by 3 years' follow-up.

\section{Preventive behaviors}

All subjects with a positive test result for HNPCC had had a colonoscopy in the 3 years after testing. These data add to the 12 -month results ${ }^{15}$ by showing that the small number of carriers who had not had a colonoscopy within 12 months of testing had had one within 3 years. Most studies that have assessed screening and surveillance behavior have shown that people usually underwent colonoscopy screening at a level appropriate to their mutation status within 1 year of testing, ${ }^{15,29,31}$ although both over- and under-screening among mutation carriers has been reported in one study, ${ }^{32}$ and one only reported data for carriers. ${ }^{33}$

The finding that only $7 \%$ of non-carriers had had a colonoscopy within the 2 years before the 3 -year follow-up gives further evidence that people are generally reassured by a negative genetic test result and are willing to discontinue intensive bowel screening. There had been some suggestion that those found to be at low risk might be reluctant to cease intensive surveillance. ${ }^{34-36}$ However, our study provides evidence to the contrary. As the effectiveness of a predictive testing program depends on appropriate screening behaviors after testing, ${ }^{13,37}$ these findings are important in showing that even over a period of 3 years, most people are following guidelines for colonoscopy screening.

The findings regarding use of FOBT in non-carriers who are in the age range in which population level screening is indicated suggest that once individuals are taken off the intensive screening program, many do not access population screening. The most likely reason for this is that, at the time of this study, bowel screening at the population level was not offered in a coordinated state-funded program despite the existence of guidelines for population screening. ${ }^{18}$ Moreover, primary health care physicians may not have been clear about the guidelines and therefore not actively offering screening. However, after a successful pilot program, population FOBT screening is now being introduced in a coordinated, government-funded program to people older than 55 years; this may well increase participation rates for all, including those from families with HNPCC. ${ }^{38}$

The proportion of female carriers reporting gynecological screening in this study was slightly higher than that reported at 12 months,${ }^{15}$ although the number of female carriers was small $(n=13)$. From these limited data, it seems that some women are following the Australian clinical practice guidelines, ${ }^{18}$ although they are less prescriptive than those for CRC screening. The reasons reported for having screening suggest that women recognized their high risk of endometrial cancer and had a 
belief in the efficacy of the screening procedures. Previous research on cancer screening ${ }^{39}$ would suggest that a family history of endometrial cancer might be associated with uptake of endometrial screening. However, the number of carriers in our study was too small to assess this relationship in a statistically meaningful way. The only other study that reported on uptake of endometrial sampling showed a similar proportion (69\%) of female carriers older than 35 years who reported having had a transvaginal ultrasound within 6 months to 8.5 years after testing. ${ }^{33}$ It was not clear why a small number of non-carriers in our study reported gynecological screening. It is possible that there was some confusion between gynecological screening for cancer and other forms of screening, such as pap smears, or transvaginal ultrasound for the purpose of cancer screening and other forms of ultrasound.

Overall, the results regarding both colorectal and gynecological screening show that, although many have adopted appropriate screening, some participants may still benefit from additional genetic counseling to clarify risk and screening recommendations, particularly among non-carriers.

In our 12-month report, ${ }^{15}$ we suggested that one of the reasons for the lack of uptake of prophylactic surgery could be that the time required to make a decision about surgery may be longer than 12 months. These data show that even within 3 years, colorectal surgery is not a chosen option for this population at risk of HNPCC. The most likely reason for this is the demonstrated efficacy of colonoscopy screening, such that surgery may not be considered necessary to prevent bowel cancer. With respect to gynecological cancers, only one article, published in early 2006, has demonstrated the efficacy of prophylactic surgery in reducing the occurrence of endometrial and ovarian cancers. ${ }^{40}$ It remains to be seen whether studies of this kind will affect decisions regarding prophylactic surgery. It is also possible that, in other health systems in which gynecological screening may not be so readily available, decisions regarding prophylactic surgery may well be different.

It is important to collect longer term data to fully document the effects of genetic testing on psychological outcomes and screening behaviors. ${ }^{28,31}$ However, there are well-known, inherent problems in long-term follow-up. For example, in our study, some participants had died from cancer (or other causes) or had developed cancer, thus becoming ineligible for the follow-up survey. Analysis of potential biases resulting from those lost to follow-up showed that the non-responders had higher mean cancer-specific distress levels at baseline than the responders, although they were similar in other respects. Therefore, the possibility that this study describes the best-case scenario for psychological outcomes of participants after 3 years cannot be excluded.

In summary, the findings from this study, the first longer term follow-up of individuals undergoing genetic testing for HNPCC, are very encouraging. The results suggest that the information provided by the genetic test result is being used in a way that is helpful to the individuals concerned and is not causing undue anxiety or distress. It will be very interesting to see whether similar results are seen in other populations or in areas with different health care systems.

\section{ACKNOWLEDGMENTS}

This research was supported by a 3-year project grant from The Cancer Council Victoria. B.M. is supported by Public Health Australia Fellowship 007079 from the National Health and Medical Research Council of Australia and J.H. is supported by National Health and Medical Research Council Career Development Award 216741.

The authors would like to acknowledge the following individuals: Ms. Rebecca Doherty, Ms. Elly Lynch, Ms. Masha Slattery, and Mr. Ivan Macciocca from the Familial Cancer Centre, Royal Melbourne Hospital and Genetic Health Services Victoria; Ms. Mary-Anne Young, Ms. Linda Warwick, Ms. Lisette Curnow, and Ms. Anna Henry from the Familial Cancer Centre, Peter MacCallum Cancer Institute, Melbourne; Dr. Jack Goldblatt, Dr. Ian Walpole, Ms. Jillian Kennedy, and Ms. Karen Harrop from Genetics Services of Western Australia; Dr. Michael Gattas, Dr. Jenny Kromberg, Ms. Julie White, and Ms. Kay Podger from Queensland Clinical Genetics Service; Prof. Allan Spigelman, Ms. Bronwyn Burgess, and Ms. Claire Groombridge from Hunter Family Cancer Service, NSW; Dr. Kathy Tucker, Ms. Monica Tucker, and Ms. Margaret Gleeson from the Hereditary Cancer Clinic, Prince of Wales Hospital, NSW; and Ms. Rosemary Warren from Public Health Genetics, Murdoch Childrens Research Institute, for assistance with patient recruitment, data collection, and the ethics application process. We are also most grateful for the valuable contribution of all the individuals who participated in this study.

\section{References}

1. Katballe N, Christensen M, Wikman FP, Orntoft TF, et al. Frequency of hereditary non-polyposis colorectal cancer in Danish colorectal cancer patients. Gut 2002;50: 43-51.

2. de la Chapelle A. The incidence of Lynch syndrome. Fam Cancer 2005;4:233-237.

3. Lynch HT, de la Chapelle A. Genetic susceptibility to non-polyposis colorectal cancer. J Med Genet 1999;36:801-818.

4. Dunlop MG, Farrington SM, Carothers AD, Wyllie AH, et al. Cancer risk associated with germline DNA mismatch repair gene mutations. Hum Mol Genet 1997;6:105110.

5. Aarnio M, Sankila R, Pukkala E, Salovaara R, et al. Cancer risk in mutation carriers of DNA-mismatch-repair genes. Int J Cancer 1999;81:214-218.

6. Van de Water NS, Jeevaratnam P, Browett PJ, Stewart SM, et al. Direct mutational analysis in a family with hereditary non-polyposis colorectal cancer. Aust NZ J Med 1994;24:682-686

7. Liu B, Parsons RE, Hamilton SR, Petersen GM, et al. hMSH2 mutations in hereditary nonpolyposis colorectal cancer kindreds. Cancer Res 1994;54:4590-4594.

8. Jenkins MA, Baglietto L, Dowty JG, Van Vliet CM, et al. Cancer risks for mismatch repair gene mutation carriers: a population-based early onset case-family study. Clin Gastroenterol Hepatol 2006;4:489-498.

9. Hampel H, Stephens JA, Pukkala E, Sankila R, et al. Cancer risk in hereditary nonpolyposis colorectal cancer syndrome: later age of onset. Gastroenterology 2005;129: 415-421.

10. Carayol J, Khlat M, Maccario J, Bonaiti-Pellie C. Hereditary non-polyposis colorectal cancer: current risks of colorectal cancer largely overestimated. J Med Genet 2002;39:335-339.

11. Jarvinen HJ, Aarnio M, Mustonen H, Aktan-Collan K, et al. Controlled 15-year trial on screening for colorectal cancer in families with hereditary nonpolyposis colorectal cancer. Gastroenterology 2000;118:829-834.

12. de Jong AE, Hendriks YM, Kleibeuker JH, de Boer SY, et al. Decrease in mortality in Lynch syndrome families because of surveillance. Gastroenterology 2006;130:665-671. 
13. Breheny N, Geelhoed E, Goldblatt J, Ee H, et al. Economic evaluation of the familial cancer programme in Western Australia: predictive genetic testing for familial adenomatous polyposis and hereditary non-polyposis colorectal carcinoma. Commun Genet 2006;9:98-106.

14. Meiser B, Collins V, Warren R, Gaff C, et al. Psychological impact of genetic testing for hereditary nonpolyposis colorectal cancer (HNPCC). Clin Genet 2004;66:502511

15. Collins V, Meiser B, Gaff C, St. John DJ, et al. Screening and preventive behaviors one year after predictive genetic testing for hereditary nonpolyposis colorectal carcinoma. Cancer 2005;104:273-281.

16. Australian Cancer Network. Guidelines on familial aspects of cancer. Sydney: Australian Cancer Network, 1999.

17. Human Genetics Society of Australasia. Guidelines for DNA presymptomatic and predictive testing for genetic disorders. Sydney: Human Genetics Society of Australasia, 2002.

18. Australian Cancer Network Colorectal Cancer Guidelines Revision Committee. Guidelines for the prevention, early detection and management of colorectal cancer. Sydney: The Cancer Council Australia and Australian Cancer Network, 2005.

19. Myers RE, Ross E, Jepson C, Wolf T, et al. Modeling adherence to colorectal cancer screening. Prev Med 1994;23:142-151.

20. Moorey S, Greer S, Watson M, Gorman C, et al. The factor structure and factor stability of the hospital anxiety and depression scale in patients with cancer. $\mathrm{Br}$ J Psychiatry 1991;158:255-259.

21. Zigmond AS, Snaith RP. The hospital anxiety and depression scale. Acta Psychiatr Scand 1983;67:361-370.

22. Marteau T, Bekker H. The development of a six-item short-form of the state scale of the state-trait anxiety inventory (STAI). Br J Clin Psychol 1992;31:301-306.

23. Michie S, French DP, Marteau TM. Predictive genetic testing: mediators and moderators of anxiety. Int J Behav Med 2002;9:309-321.

24. Horowitz M, Wilner N, Alvarez W. Impact of event scale: a measure of subjective stress. Psychosom Med 1979;41:209-218.

25. Thewes B, Meiser B, Hickie IB. Psychometric properties of the Impact of Event Scale amongst women at increased risk for hereditary breast cancer. Psychooncology 2001; 10:459-468.

26. Goldstein H. Multilevel statistical models. London: Arnold, 1995

27. Davison A, Hinkley D. Bootstrap methods and their application. Cambridge: Cambridge University Press, 1997.
28. Aktan-Collan K, Haukkala A, Mecklin J-P, Uutela A, et al. Psychological consequences of predictive genetic testing for hereditary non-polyposis colorectal cancer (HNPCC): a prospective follow-up study. Int J Cancer 2001;93:608-611.

29. Claes E, Denayer L, Evers-Kiebooms G, Boogaerts A, et al. Predictive testing for hereditary nonpolyposis colorectal cancer: subjective perception regarding colorectal and endometrial cancer, distress, and health-related behavior at one year posttest. Genet Test 2005;9:54-65.

30. Gritz ER, Peterson SK, Vernon SW, Marani SK, et al. Psychological impact of genetic testing for hereditary nonpolyposis colorectal cancer. J Clin Oncol 2005;23:19021910 .

31. Halbert $\mathrm{CH}$, Lynch $\mathrm{H}$, Lynch J, Main D, et al. Colon cancer screening practices following genetic testing for hereditary nonpolyposis colon cancer (HNPCC) mutations. Arch Intern Med 2004;164:1881-1887.

32. Hadley DW, Jenkins JF, Dimond E, de Carvalho M, et al. Colon cancer screening practices after genetic counseling and testing for hereditary nonpolyposis colorectal cancer. J Clin Oncol 2004;22:39-44.

33. Ponz de Leon M, Benatti P, Di Gregorio C, Pedroni M, et al. Genetic testing among high-risk individuals in families with hereditary nonpolyposis colorectal cancer. $\mathrm{Br}$ J Cancer 2004;90:882-887.

34. Michie S, Weinman J, Miller J, Collins V, et al. Predictive genetic testing: high risk expectations in the face of low risk information. J Behav Med 2002;25:33-50.

35. Michie S, Smith JA, Senior V, Marteau TM. Understanding why negative genetic test results sometimes fail to reassure. Am J Med Genet 2003;119A:340-347.

36. Bleiker EMA, Menko FH, Taal BG, Kluijt I, et al. Experience of discharge from colonoscopy of mutation negative HNPCC family members. J Med Genet 2003;40:e55.

37. Stanley AJ, Gaff CL, Aittomaki AK, Fabre L, et al. Value of predictive genetic testing in management of hereditary non-polyposis colorectal cancer (HNPCC). Med J Aust 2000;172:313-316.

38. Australian Government Department of Health and Ageing. National Bowel Cancer Screening Program. Available at http://www.cancerscreening.gov.au/ internet/screening/ publishing.nsf/Content/bowel-about. Accessed January 17, 2007.

39. Madlensky L, Esplen MJ, Gallinger S, McLaughlin JR, et al. Relatives of colorecta cancer patients: factors associated with screening behavior. Am J Prev Med 2003;25: 187-194.

40. Schmeler KM, Lynch HT, Chen LM, Munsell MF, et al. Prophylactic surgery to reduce the risk of gynecologic cancers in the Lynch syndrome. N Engl J Med 2006; 354:261-269. 